\section{P083/023 NOVEL SUBCLASS OF NON-CLASSICAL MONOCYTES ARE CRITICAL FOR INFLAMMATION}

${ }^{1} \mathrm{AB}$ Montgomery*, 1,2PJ Homan, 'D Winter, 'HR Perlman. 'Northwestern University; ${ }^{2}$ Rheumatology, Chicago, USA

10.1136/annrheumdis-2018-EWRR2019.72

Career situation of first and presenting author Post-doctoral fellow.

Introduction Monocytes in mice are distinguishable by expression of Ly6c. Ly6 $\mathrm{c}^{\text {hi }}$ (classical) monocytes are associated with pro-inflammatory responses, while Lyc6 ${ }^{\text {lo }}$ (non-classical) are involved in patrolling endothelial membranes. We have previously shown that depletion of monocytes prevents serum transfer induced arthritis (STIA) in mice, and that Lyc6 ${ }^{\text {lo }}$ monocytes are the critical population.

Objectives We aim to contrast Ly6 $\mathrm{c}^{\mathrm{lo}}$ monocytes from the circulation, lining vessels, and tissue to determine their involvement in inflammation.

Methods Female 8-10 week old NR4A1 $1^{-/}$, CX3CR $1^{\text {ERCre. }}$ zsGFP, and $\mathrm{C} 57 \mathrm{Bl} / 6$ mice were used in all studies. CX3CR1 ${ }^{\text {ERCre.zsGFP }}$ were utilized for cell tracking studies and joint shielded bone marrow chimeras via administration of tamoxifen (tam). Intravascular monocytes were identified by I.V. anti-CD45 labeling before perfusion. STIA was induced via I.V. KBxN sera. Cell populations were quantified by flow cytometry and FACS sorted for RNA-seq. Monocytes were identified $\mathrm{CD}_{4}{ }^{+} \mathrm{CD}_{11 b^{+}} \mathrm{Ly}_{6 G^{-}}$TIM4 $\mathrm{CD}^{-} 4^{-} \mathrm{Ly} \mathrm{c}^{\mathrm{lo}}$ and subdivided into intravascular (labeled, $\mathrm{CD} 43^{+}$), trans-vascular (labeled $\mathrm{CD}^{\circ} 3^{-}$) and extravascular (no label).

Results NR4A $1^{-/-}$mice retain only $5 \%$ of circulating Ly $6 \mathrm{c}^{\mathrm{lo}}$ monocytes but all joint $\mathrm{Ly}_{6} \mathrm{c}^{\mathrm{lo}}$ cells. STIA was comparable in $\mathrm{NR}_{4} \mathrm{~A}^{-/-}$and $\mathrm{C} 57 \mathrm{Bl} / 6$ mice suggesting circulating Ly $6 \mathrm{c}^{\text {lo }}$ are redundant. Transcriptional profiling of $\mathrm{Ly}_{6} \mathrm{c}^{\mathrm{lo}}$ cells identified distinct pathways enriched in upregulated genes between $\mathrm{Ly}_{6 \mathrm{c}} \mathrm{lo}^{\mathrm{o}}$ from joint and blood. In the joint we identified three populations of $\mathrm{Ly} 6 \mathrm{C}^{\mathrm{lo}}$ monocytes: extravascular unlabeled cells, labeled trans-vascular cells, and labeled intravascular cells adherent to endothelium. Mice given tam D8 of gestation had $\mathrm{GFP}^{+}$microglia only, whereas D15 tam induced $\mathrm{GFP}^{+}$synovial macrophages and unlabeled Ly $6 c^{\text {lo }}$ monocytes. Both labeled Ly $6 c^{\text {lo }}$ populations were $\mathrm{GFP}^{-}$, indicating unlabeled and unlabeled Ly $6 \mathrm{c}^{\mathrm{lo}}$ arise from different progenitors. This was confirmed by bone marrow chimera studies showing labeled Ly $6 \mathrm{c}^{\mathrm{lo}}$ cells are replenished from blood monocytes. Clodronate loaded liposomes depleted labeled $\mathrm{CD}_{4} 3^{+}$cells but did not affect $\mathrm{CD}^{-} 3^{-}$cells or unlabeled cells. With with our previous finding that clo-lip prevents STIA, these suggest adherent $\mathrm{CD}_{4} 3^{+} \mathrm{Ly}_{6 \mathrm{c}^{\text {lo }}}$ cells are essential. This is supported by the finding that labeled Ly $6 \mathrm{c}^{\mathrm{lo}}$ monocytes expand rapidly during the first 1 hour of STIA. Adherent $\mathrm{CD}_{4} 3^{+}$cells expand especially rapidly, increasing in population size by $30 x$.

Conclusions We have identified and described two previously uncharacterized populations of $\mathrm{Ly}_{6} \mathrm{c}^{\mathrm{lo}}$ cells in the joint- intravascular adherent and trans-vascular which have distinct origins and phenotype from both extravascular and circulating Ly $6 \mathrm{c}^{\mathrm{lo}}$. The findings presented here strongly suggest adherent Ly $6 \mathrm{c}^{\text {lo }}$ monocytes are a key effector cell in inflammatory arthritis.

Disclosure of Interest None declared.

\section{P084 SALIVARY GLAND EPITHELIAL CELLS FROM SJÖGRENS' PATIENTS INCREASE B LYMPHOCYTES SURVIVAL AND ACTIVATION}

1,2E Rivière*, 'J Pascaud, 'A Virone, 'B Ly, ${ }^{1} \mathrm{~A}$ Paoletti, ${ }^{1} \mathrm{~J}$ Rohmer, 'S Bitoun,

${ }^{1,3} \mathrm{G}$ Nocturne, ${ }^{1,3} \mathrm{X}$ Mariette. ${ }^{1}$ IMVA, CEA-Université Paris Sud-INSERM U1184, Le Kremlin Bicêtre; ${ }^{2}$ Fondation Arthritis Courtin, Paris; ${ }^{3}$ AP-HP, Paris-Sud, Le Kremlin Bicêtre, France

\subsection{6/annrheumdis-2018-EWRR2019.73}

Career situation of first and presenting author Student for a master or a $\mathrm{PhD}$.

Introduction Primary Sjögren's syndrome (pSS) is a chronic autoimmune disorder characterized by lymphocytic infiltrates and destruction of the salivary glands (SG). Several lines of evidence support the hypothesis that SG epithelial cells (SGECs) are not only the target of autoimmunity in pSS but may also play a role for its initiation and maintenance.

Objectives To study the survival and the activation of B lymphocytes cocultured with SGECs from pSS patients compared to controls.

Methods Primary cultures of SGECS were established from minor SG biopsies. Patients had pSS according to 2016 EULAR/ACR criteria and controls had sicca symptoms without any antibodies and with normal SG biopsies. The coculture involved B lymphocytes isolated by CD19 magnetic bead positive selection from healthy donors' blood (purity >80\%). Several conditions of stimulation were tested: IFNa $2400 \mathrm{U} / \mathrm{mL}$, IFNg $5 \mathrm{ng} / \mathrm{mL}$, Poly(IC) $10 \mu \mathrm{g} / \mathrm{mL}$ or $30 \mu \mathrm{g} / \mathrm{mL}$. After 5 days, the viability, the activation (CD38) and the differentiation (CD27) of B lymphocytes were assessed by flow cytometry. Mann-Whitney (unpaired data) and Wilcoxon (paired data) were used for statistical analysis.

Results A significant increase of B lymphocytes survival was observed when cocultured with SGECs compared to B lymphocytes cultured alone, in all conditions of stimulation $(p<0.05)$. The survival of B lymphocytes (percentage of alive cocultured B lymphocytes-percentage of alive cultured alone B lymphocytes) was increased when the cocultures were performed with SGECs from pSS patients $(n=5)$ compared to SGECs from controls $(n=5)$, in all conditions of stimulation $(p<0.05)$, except IFNg. Moreover, there was a trend for an increase of B lymphocytes activation, assessed by higher percentages of CD38+ B lymphocytes when the cocultures were performed with SGECs from pSS patients compared to SGECs from controls. This difference was statistically significant $(p<0.05)$ in the condition stimulated with TLR3 agonist (Poly(IC) $10 \mu / \mathrm{mL}$ ). The percentage of CD27 + B lymphocytes was not affected by the cocultures and no difference between pSS and controls SGECs was observed.

Conclusions This coculture model showed a differential effect of SGECs from pSS compared to controls on B lymphocytes survival. Interestingly, there was also a trend for a higher activation level of B lymphocytes when cocultured with SGECs from pSS compared to controls. These results suggest that SGECs could play a major role in pSS pathophysiology through B lymphocytes support and activation.

Disclosure of Interest None declared.

\section{P085 ACTIVATED MEMORY T CELLS PRODUCE LIGANDS THAT CAUSE NF-KB-DEPENDENT INFLAMMATORY ACTIVATION OF THE ENDOTHELIUM}

KC Jeucken, JP van Hamburg, SW Tas*. Experimental Immunology/Clinical Immunology and Rheumatology, Amsterdam UMC, Amsterdam, Netherlands

10.1136/annrheumdis-2018-EWRR2019.74 
Career situation of first and presenting author Student for a master or a $\mathrm{PhD}$.

Introduction Endothelial cells (EC) are important contributors to inflammation via expression of inflammatory mediators, including chemokines and adhesion molecules. Production of these inflammatory mediators can be induced via canonical and NF- $\kappa \mathrm{B}$-inducing kinase (NIK)-dependent noncanonical NF$\kappa \mathrm{B}$ signalling. The ligands activating these pathways are well studied, but less is known about the cells producing ligands that can activate NF- $\mathrm{BB}$ signalling in EC.

Objectives To study the effects of soluble factors produced by activated memory $\mathrm{T}\left(\mathrm{T}_{\mathrm{m}}\right)$ cells on NF- $\kappa \mathrm{B}$ dependent inflammatory activation of EC.

Methods $\mathrm{CD}^{+} \mathrm{CD}_{45 \mathrm{RO}^{+}}$Memory $\mathrm{T}$ cells were isolated from healthy PBMC using MACS sorting and cultured in medium containing anti-CD3 and anti-CD28 for 72 hour, after which supernatant was harvest. Human umbilical cord EC (HUVEC) were stimulated with $50 \% \mathrm{~T}_{\mathrm{m}}$ supernatant $\left(\mathrm{T}_{\mathrm{m}}\right.$ sup). After 72 hour of $\mathrm{T}_{\mathrm{m}}$ sup stimulation HUVEC protein and RNA was harvest and $\mathrm{NF}-\mathrm{\kappa B}$ signalling and downstream expression of inflammatory mediators was analysed using qPCR and Western Blot. Culture supernatant was analysed by ELISA to detect presence of inflammatory mediators. To repress canonical NF- $\kappa \mathrm{B}$ signalling an inhibitor of IKK $\beta$ (iIKK $\beta$ ) was used and to repress NIK-dependent NF$\kappa \mathrm{B}$ signaling an inhibitor of NIK (iNIK) was used.

Results Stimulation with $\mathrm{T}_{\mathrm{m}}$ sup led to activation of both canonical NF- $\kappa \mathrm{B}$ signalling, indicated by increased levels of phosphorylated ( $\mathrm{p})-\mathrm{I} \kappa \mathrm{B} \alpha$, and noncanonical NF- $\mathrm{B}$ signalling, indicated by increased p100 to p52 processing. HUVEC stimulated with $T_{m}$ sup had increased mRNA levels of all tested inflammatory mediators compared to non-treated cells. Gene expression of chemokines (CXCL1, CXCL5, IL6, IL8 and GM-CSF) after $T_{m}$ sup stimulation was significantly reduced after treatment with iIKK $\beta$ and to a lesser, but still significant, extent after treatment with iNIK. Interestingly, treatment with iIKK $\beta$ also led to a reduction in mRNA levels of the adhesion molecules VCAM-1 and ICAM-1, while this effect was minimal after iNIK treatment. In addition, treatment with either IKK $\beta$ or iNIK led to a significant reduction in CXCL5 in the culture supernatant of HUVEC stimulated with $\mathrm{T}_{\mathrm{m}}$ sup.

Conclusions This study provides new insights into the cellular interactions leading to production of inflammatory mediators by EC. Our findings demonstrate that activated $T_{m}$ cells fac-

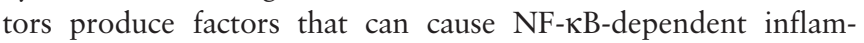
matory activation of EC. Targeting canonical NF- $\kappa B$ signaling

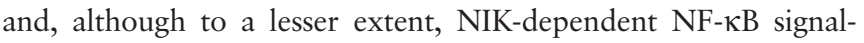
ing reduces inflammatory activation of the endothelium.

Disclosure of Interest None declared.

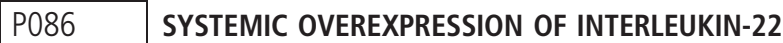 INDUCES EXPRESSION OF THE NEGATIVE IMMUNE- REGULATOR SOCS3 AND POTENTLY REDUCES COLLAGEN-INDUCED ARTHRITIS IN MICE}

J Aarts*, D Roeleveld, B Walgreen, M Helsen, E Vitters, F Van de Loo, P Van Lent, W Van den Berg, P Van der Kraan, M Koenders. Experimental Rheumatology, Radboud University Medical Center, Nijmegen, Netherlands

\subsection{6/annrheumdis-2018-EWRR2019.75}

Career situation of first and presenting author Student for a master or a $\mathrm{PhD}$.

Introduction High interleukin-22 (IL-22) levels are detected in serum and synovial fluid of rheumatoid arthritis (RA) patients and have been shown to positively correlate with disease activity markers and more erosive disease. The role of IL-22 in autoimmunity and inflammation appears to be greatly contradictory, being both pro- and anti-inflammatory. Especially the antiinflammatory properties of IL-22 are not well understood. We aimed to investigate the anti-inflammatory and immune-suppressive effect of IL-22 during experimental arthritis.

Methods Collagen-induced arthritis was induced in DBA1 mice by immunization and booster with bovine collagen type II (CII). After booster, but before arthritis onset, IL-22 was overexpressed either locally or systemically using an adenoviral construct (AdIL-22) or Luciferase as control (AdLuc). $1 \times$ $10^{7}$ plaque-forming units (PFU) of the adenoviruses were injected intra-articularly for local overexpression, or $3 \times$ $10^{8}$ PFU was injected intravenously for systemic overexpression in immunized mice, and mice were sacrificed 10 days later. Macroscopic scoring and histological analysis was performed, and mRNA expression and protein production of various pro- and anti-inflammatory mediators was determined in synovial tissue, spleen, and serum.

Results Local overexpression of IL-22 by injection of AdIL-22 in the knee joint of CII-immunized mice resulted in an unaltered arthritis incidence and severity as compared to the control virus AdLuc. Accordingly, no changes in mRNA expression or protein production were observed in CIA mice locally overexpressing IL-22. In contrast, systemic overexpression of IL-22 potently reduced disease incidence and severity, which was also confirmed by histological analysis. Systemic levels of IL-1 $\beta$, IL-17, GM-CSF and MCP1 were unaltered in mice overexpressing IL-22 systemically. However, these mice showed significantly lower serum levels of IFN $\gamma, \mathrm{TNF} \alpha$, MIP1 $\alpha$, and IL-10. Interestingly, the significantly enhanced splenic SOCS3 expression was found to negatively correlate to serum TNF $\alpha$ and MIP1 $\alpha$ levels, which is in line with our hypothesis that that the observed reduction in the cytokine levels is mediated in a SOCS3-dependent manner.

Conclusions With this study, we revealed clear anti-inflammatory effects of IL-22 overexpression during collagen-induced arthritis, which are completely dependent on the systemic route of administration. Additionally, we were the first to show that this protective effect of IL-22 during experimental arthritis is likely orchestrated via up-regulation of the negative regulator SOCS3.

Disclosure of Interest None declared.

\section{P087 THE ANTI-INFLAMMATORY CYTOKINE INTERLEUKIN 37 IS AN ENDOGENOUS INHIBITOR OF TRAINED IMMUNITY}

${ }^{1,2,3} \mathrm{G}$ Cavalli*, ${ }^{4} \mathrm{M}$ Gresnigt, ${ }^{5} \mathrm{~T}$ Nemkov, ${ }^{2} \mathrm{R}$ Arts, ${ }^{5} \mathrm{~A}$ D'Alessandro, ${ }^{6} \mathrm{~S}$ Giugliano, ${ }^{7} \mathrm{E}$ Eisenmensser, ${ }^{1} \mathrm{~L}$ Dagna, ${ }^{2} \mathrm{~L}$ Joosten, ${ }^{2} \mathrm{M}$ Netea, ${ }^{2,3} \mathrm{C}$ Dinarello. ${ }^{1}$ Unit of Immunology, Rheumatology, Allergy and Rare Diseases (UniRAR), Vita-Salute San Raffaele University, Milan, Italy; 'Department of Medicine, Radboud University Medical Center, Nijmegen, Netherlands; ${ }^{3}$ Department of Medicine, University of Colorado Denver, Aurora, CO, USA; ${ }^{4}$ Microbial Pathogenicity Mechanisms, Leibniz Institute for Natural Product Research and Infection Biology, Jena, Germany; ${ }^{5}$ Department of Biochemistry and Molecular Genetics, University of Colorado Denver, Aurora, CO, USA; ${ }^{6}$ Mucosal Immunology and Microbiota Unit, Humanitas University, Milan; 'Department of Biochemistry and Molecular Genetics, University of Colorado Denver, Aurora, CO, Italy

\subsection{6/annrheumdis-2018-EWRR2019.76}

Career situation of first and presenting author Assistant. Introduction Trained immunity (TI) is a de-facto innate immune memory program induced in monocytes/macrophages 